\title{
Electrochemical Bridging of Conducting Polymers at the Percolation Threshold for Highly Sensitive Chemiresistors
}

\author{
Krishnan Murugappan ${ }^{1 *}$, Ben Armitage ${ }^{1}$, Merel Lefferts ${ }^{1}$, Tabitha Jones ${ }^{1}$, Martin Castell ${ }^{1}$ \\ ${ }^{1}$ Department of Materials, University of Oxford, Parks Road, Oxford OX1 3PH, \\ krishnan.murugappan@materials.ox.ac.uk
}

\begin{abstract}
Interdigitated Electrodes (IDEs) are electrodes where two opposing metal electrodes are deposited on an inert substrate with a 'comb' like structure. IDEs are increasingly being investigated for their use in electrochemical sensors especially in chemiresistors as they exhibit high sensitivity due to their favourable geometry. The fingers in the two combs are separated by a distance usually in the $\mu \mathrm{m}$ range. In this work a thin layer of gold is evaporated onto the IDEs and then annealed at high temperatures to form gold nano islands through the process of 'dewetting' thereby converting the micro gaps in between the fingers to nano gaps, which further improves their geometry. These nanogaps are then bridged by conducting polymers at the 'electrical percolation region'. In a chemiresistor, the conducting polymer/sensing layer is usually deposited as a thin film on the IDEs, which is termed as the 'thin film region'. However by operating the IDE in the 'electrical percolation region' an enhancement of sensitivity and response times can be achieved.[1] We have recently shown how conducting polymer bridges can be formed in the percolation region in-between micro gaps [2] and will be extending this to nano-gaps. In this work PEDOT is electrochemically deposited between the nanogaps and then the sensors in the percolation region and thin film region are compared. It will be shown that much higher sensitivities and response times are obtained for sensors operating in the percolation region for ammonia gas detection.
\end{abstract}

Key words: chemiresistors, conducting polymers, electrochemical deposition, percolation threshold, gas sensors
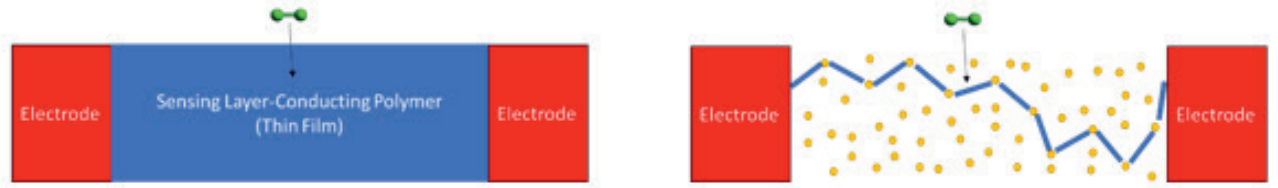

Fig.1. A thin film chemiresistor is depicted on the left and a chemiresistor in the percolation region containing a network of gold nanoparticles and conducting polymers is shown on the right.

\section{References}

[1] Sauerwald, T. and Russ, S. in Gas Sensing Fundamentals (eds Claus-Dieter Kohl \& Thorsten Wagner) 247-278 (Springer Berlin Heidelberg, 2014).

[2] Murugappan, K, Castell, M. R. Bridging electrode gaps with conducting polymers around the electrical percolation threshold. Electrochemistry Communications 87, 40-43, (2018); DOI:10.1016/j.elecom.2017.12.019. 